\title{
The RING ubiquitin E3 RNF114 interacts with A20 and modulates NF- $\kappa$ B activity and T-cell activation
}

\author{
This article has been corrected since Online Publication and a corrigendum has also been published
}

MS Rodriguez ${ }^{1}$, I Egaña ${ }^{2}$, F Lopitz-Otsoa ${ }^{2}$, F Aillet ${ }^{1}$, MP Lopez-Mato ${ }^{3}$, A Dorronsoro ${ }^{4}$, S Lobato-Gil ${ }^{1}$, JD Sutherland ${ }^{2}$, R Barrio ${ }^{2}$, C Trigueros ${ }^{4}$ and V Lang ${ }^{*, 1}$

Accurate regulation of nuclear factor- $\kappa \mathrm{B}$ (NF- $\kappa \mathrm{B}$ ) activity is crucial to prevent a variety of disorders including immune and inflammatory diseases. Active NF- $\kappa$ B promotes $I \kappa B \alpha$ and $A 20$ expression, important negative regulatory molecules that control the NF- $\kappa$ B response. In this study, using two-hybrid screening we identify the RING-type zinc-finger protein 114 (RNF114) as an A20-interacting factor. RNF114 interacts with A20 in T cells and modulates A20 ubiquitylation. RNF114 acts as negative regulator of NF- $\kappa$ B-dependent transcription, not only by stabilizing the A20 protein but also I $\kappa \mathrm{B} \alpha$. Importantly, we demonstrate that in T cells, the effect of RNF114 is linked to the modulation of T-cell activation and apoptosis but is independent of cell cycle regulation. Altogether, our data indicate that RNF114 is a new partner of A2O involved in the regulation of NF- $\kappa$ B activity that contributes to the control of signaling pathways modulating $T$ cell-mediated immune response.

Cell Death and Disease (2014) 5, e1399; doi:10.1038/cddis.2014.366; published online 28 August 2014

Nuclear factor $-\kappa \mathrm{B}(\mathrm{NF}-\kappa \mathrm{B})$ is a principal transcriptional regulator playing a pivotal part in innate and adaptive immunity, inflammation, development, cell proliferation and survival. ${ }^{1,2}$ Defects in the regulation of NF- $\kappa \mathrm{B}$-dependent gene expression contribute to a variety of diseases, including inflammatory and autoimmune diseases, neurological disorders and cancer. ${ }^{3-5}$ Therefore, activation of NF- $\kappa \mathrm{B}$ is tightly regulated by several NF- $\kappa \mathrm{B}$ target genes such as $\mathrm{I}_{\kappa} \mathrm{B} \alpha, \mathrm{A} 20$ and CYLD that function as inhibitors in a negative feedback loop. ${ }^{6-10}$ A20 (also known as TNFAIP3) is a cytoplasmic zincfinger protein that was originally identified as a tumor necrosis factor (TNF)-inducible protein and it has been characterized as a dual inhibitor of NF- $\kappa$ B activation and cell death. ${ }^{11}$ In most cell types, basal A20 expression is very low but its transcription is rapidly induced upon $\mathrm{NF}-\kappa \mathrm{B}$ activation. The essential role of $\mathrm{A} 20$ in the regulation of $\mathrm{NF}-\kappa \mathrm{B}$ and apoptotic signaling was clearly demonstrated with the generation of a complete A20 knockout mouse. ${ }^{12,13}$ Mice deficient for A20 are hypersensitive to TNF and die prematurely because of severe multiorgan inflammation and cachexia. ${ }^{12}$ However, the antiapoptotic function of A20 is not a general feature, as A20 only protects some cell types from specific death-inducing agents. ${ }^{14}$ Protein ubiquitylation plays an important role in the regulation of $\mathrm{NF}-\kappa \mathrm{B}$ pathway, not only by controlling the stability of factors integrating this signaling cascade but also their activity. Little is known about the molecular mechanisms that regulate ubiquitin-editing and NF- $\kappa \mathrm{B}$-inhibitory function of A20. Up to date, two enzymatic activities have been associated to A20, a C-terminal ubiquitin ligase and a $\mathrm{N}$-terminal de-ubiquitylating activity, acting on targets such as RIP and promoting their degradation. ${ }^{15}$ A number of A20 interacting proteins including TAX1BP1, Itch and RING-type zinc-finger protein 111 (RNF11) are known to be required for A20 to terminate NF- $\kappa$ B signaling. ${ }^{16-18}$ Interestingly, the expression, biological activities and mechanism of action of A20 are likely dependent on the cellular context as well as the stimulus involved. ${ }^{14}$ Indeed, in lymphoid cells, A20 is constitutively expressed and its expression is reduced because of activation of the paracaspase MALT1 after T-cell receptor (TCR) stimulation as well as to proteasome degradation. ${ }^{19}$ In addition, in mesenchymal stromal cells, we have recently demonstrated that $\mathrm{A} 20$ is constitutively expressed and its expression is reduced after TNF $\alpha$ stimulation because of its proteasome-induced degradation. ${ }^{20}$

In humans, polymorphisms within the $\mathrm{A} 20$ genomic region predispose individuals to autoimmune diseases such as systemic lupus erythematous, Crohn's disease and psoriasis. ${ }^{21}$ To identify new psoriasis susceptibility loci, a genome-wide association study (GWAS) of 1409 psoriasis patients and 1436 controls was carried out. ${ }^{22}$ Next to single-nucleotide polymorphisms (SNPs) in genes involved in IL-23 signaling, loci including A20, ABIN-1 (also known as TNFAIP3-interacting protein 1 (TNIP1)) and RNF114 showed strong association with psoriasis. ${ }^{22,23}$

RNF114 belongs to a recently defined family of RING (really interesting new gene) domain E3 ubiquitin ligases, characterized by the presence of three zinc-fingers and one ubiquitin interacting motif (UIM). ${ }^{24,25}$ RNF114, also known as zincfinger protein 313 (ZNF313), efficiently binds K48- and K63linked polyubiquitin chains in vitro and in vivo and possess an E3 ubiquitin ligase activity. RNF114 is a soluble cytosolic protein that can be induced by interferons and synthetic

\footnotetext{
${ }^{1}$ Cancer Unit, Inbiomed, San Sebastian, Gipuzkoa, Spain; ${ }^{2} \mathrm{CIC}$ bioGUNE, Derio, Bizkaia, Spain; ${ }^{3}$ Cytometry and Advanced Optical Microscopy Core Facility, Inbiomed, San Sebastian, Gipuzkoa, Spain and ${ }^{4}$ Hematological Diseases, Inbiomed, San Sebastian, Gipuzkoa, Spain

*Corresponding author: V Lang, Fundación Inbiomed, Ubiquitylation and Cancer Molecular Biology Laboratory, Paseo Mikeletegi 81, San Sebastian 20009, Gipuzkoa, Spain. Tel: +34 943309 064; Fax: +34 943308 222; E-mail: vlang@ @inbiomed.org

Abbreviations: RNF, RING finger protein; RING, really interesting new gene; ZNF, zinc-finger protein; GWAS, genome-wide association study; SNP, single-nucleotide polymorphism; TNF, tumor necrosis factor; TCR, T-cell receptor; UIM, ubiquitin-interacting motif

Received 03.4.14; revised 20.6.14; accepted 03.7.14; Edited by H-U Simon
} 
dsRNA. Real-time PCR analysis demonstrated that RNF114 is clearly expressed in disease-relevant cell types, including CD4 + T lymphocytes, dendritic cells and skin, and also in testis, pancreas, kidney and spleen, indicating that the activity of the RNF114 protein is unlikely to be restricted to the immune system. ${ }^{26,27}$ Recently, it was observed that RNF114 has a mitogenic function and that its deregulation can disturb cell cycle control mechanism and thus influence cellular stress response. RNF114 expression is reduced at the $\mathrm{G} 1$ phase but increased at the $S$ and G2/M transition, suggesting that its elevation may drive a $\mathrm{G} 1$ to $S$ transition of the cell cycle. ${ }^{28}$ Using a two-hybrid approach we found that RNF114 was able to interact with A20. Therefore, the goal of this work was to determine the role of this interaction on the stability and activity of $\mathrm{A} 20$ and to explore its impact on the regulation of $\mathrm{NF}-\kappa \mathrm{B}$-dependent functions.

\section{Results}

RNF114 interacts with A20. To find new A20 interacting proteins, a yeast two-hybrid screening was performed using human thymocytes (CD4 + CD8 + ) cDNA library and a fulllength form of A20 (Hybrigenics, Paris, France). In this screening, three of the proteins found, A20 itself, ABIN-1 and 14-3-3, were already described as A20 interacting proteins. ${ }^{29-31}$ The RING finger protein RNF114 was identified as a novel interacting protein. This interaction was confirmed using different approaches. First, a pull-down experiment using GST-A20 or GST fusion proteins and lysates of human embryonic kidney 293 (HEK293) cells overexpressing FLAGRNF114 or FLAG-A20 (Figure 1a) was performed as well as co-immunoprecipitations assays using HEK293 cells transfected with FLAG-A20WT in the presence or not of AU5RNF114 (Figure 1b). The immunoprecipitation experiment with anti-AU5 antibody showed a clear interaction between FLAG-A20WT and AU5-RNF114 only when both proteins were present. Signal was never detected in the immunoprecipitation control, indicating that this interaction was specific (Figure 1b). Those controls were included only in the first figure to simplify the rest of the figures. TNF $\alpha$ stimulation stabilizes FLAG-A20WT, favoring its interaction with AU5-RNF114 (Figure 1c).

To define which part of $\mathrm{A} 20$ was involved in its interaction with RNF114, different constructs of A20 were made. In the first experiment, we observed that the C-terminal part of A20 (390-790), containing the E3 ligase domain, was involved in its interaction with RNF114 (Figures 1d and e). To better define the domain of interaction, truncated forms of the $\mathrm{C}$-terminal part were made. Altogether, the results shown in the Figure 1e demonstrate that zinc-fingers 4, 5, 6 and 7 of A20 are contributing to create a solid interaction with RNF114.

Finally, to further confirm the association between the two proteins, we checked their interaction at the endogenous level in the absence of any exogenous expression. As A20 is expressed at basal conditions in $\mathrm{T}$ cells, we decided to evaluate the interaction between these two proteins in Jurkat $\mathrm{T}$ cells by doing a co-immunoprecipitation experiment using anti-A20- or anti-RNF114-specific antibodies. We confirmed a

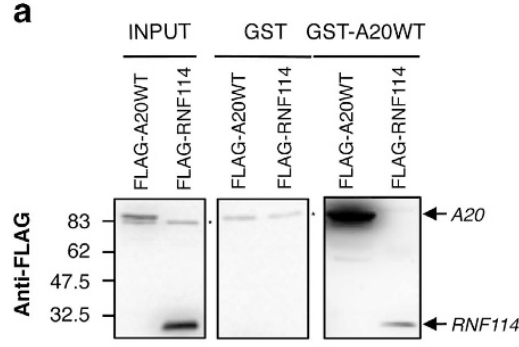

d

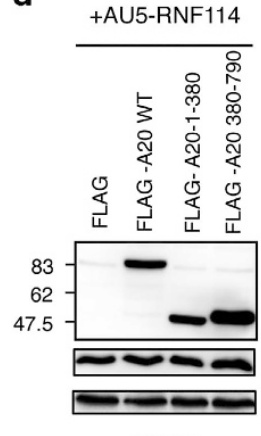

INPUT

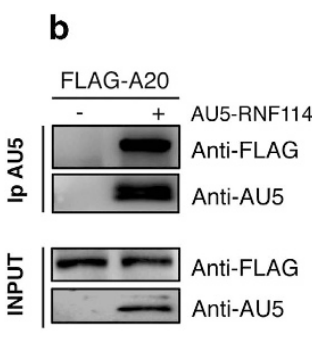

C
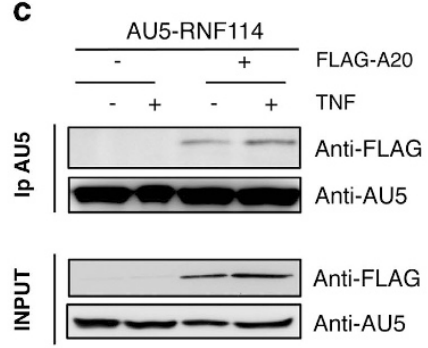

e

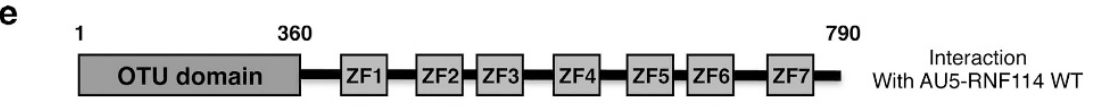

$1-790 \longrightarrow++$

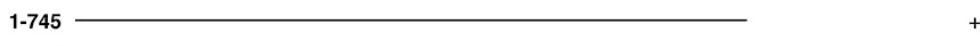

$1-686 \longrightarrow+$

$1-636-+1 /$

$1-380 \longrightarrow$

380-790 + + +

580-790

Figure 1 RNF114/ZNF313 interacts with A20. (a) Pull-down experiment using GST-A20 or GST fusion proteins and lysates of HEK293 cells transfected with FLAGA2OWT or FLAG-RNF114 is shown ( ${ }^{*}$ indicates unspecific band). (b) HEK293 cells were transfected with FLAG-A20WT and when indicated with AU5-RNF114. AU5-RNF114 immunoprecipitation was used to confirm the interaction with FLAG-A20. (c) HEK293 cells were transfected with AU5-RNF114 and FLAG-A20WT as indicated. Cells were treated with TNF $\alpha$ for $20 \mathrm{~min}$ and lysates were submitted to anti-AU5 immunoprecipitation (d) HEK293 cells were transfected with different forms of FLAG-A20 (WT, N-terminal: 1-390, C-terminal: 390-790) and AU5-RNF114 to determine which domains were involved in the interaction between A20 and RNF114. (e) Different constructs of A20 were prepared to define its interaction domain with RNF114. Results of immunoprecipitation experiments are shown. The symbol '- ' indicates no interaction and ' + ' indicates interaction 
the association between these two proteins in reciprocal experiments, even if the interaction was more obvious when the anti-RNF114 antibody was used to co-immunoprecipitate A20 (Figure 2a). This result suggests that only a fraction of A20 is associated to RNF114. However, we cannot exclude that those differences reflect the capacity of each antibody to recognize and bind these interacting molecules (Figure 2a). We checked whether the interaction between these two proteins was modified after stimulation. For that purpose, Jurkat T cells were stimulated as indicated with TNF $\alpha$ or CD3/ CD28 antibodies. We observed that the association increased after TNF $\alpha$ stimulation (Figure $2 b$ ), likely as a consequence of an increase in A20 levels after such stimuli. Interestingly, after TCR stimulation, we observed an increase in A20-RNF114 interaction and also a striking modification of A20 molecular weight associated with RNF114 (Figure 2b). These results indicate that under these stimulation conditions, the fraction of A20 able to interact with RNF114 was post-translationally modified. This modified form of A20 is not detectable in the whole lysate extract (INPUT) or after A20 immunoprecipitation (data not shown), supporting the notion that this modified form of A20 specifically bound to RNF114 is a small fraction of the total $A 20$ protein pool. According to the shift in molecular weight of the modified A20, the main band could correspond to modification by a member of the ubiquitin family rather than phosphorylation, which will be more difficult to resolve on a $10 \%$ polyacrylamide gel. Furthermore, after CD3/CD28 stimulation we also observed multiple slow migrating forms of $\mathrm{A} 20$, disposed in a pattern that is more typical of polyubiquitylation (Figure 2b). However, treatment with the proteasome inhibitor MG132 did not affect the amount or the accumulation of high-molecular-weight forms of A20 (data not shown). Altogether, the different experiments presented in these figures clearly demonstrate that RNF114 specifically interacts with $\mathrm{A} 20$, and perhaps more specifically with a modified fraction of total A20.

Effect of RNF114 on A20 ubiquitylation. RNF114 belongs to a novel family of ubiquitin ligases with zinc-fingers and an ubiquitin-binding domain, like the T-cell regulator RNF125/ TRAC-1. ${ }^{24,25}$ Therefore, we investigated whether RNF114 could promote A20 modification by coexpressing in HEK293 cells His6-ubiquitin and FLAG-A20 in the presence or not of AU5-RNF114. As shown in Figure 3a, A20 is modified with ubiquitin in the absence of AU5-RNF114; however, ubiquitylated A20 significantly increased when RNF114 is expressed. In addition, we can observe than RNF114 increases the expression level of $A 20$ in the absence of His6-ubiquitin (Input, anti-FLAG; Figure 3a). Ubiquitylation of RNF114 itself can also be seen under these conditions

a

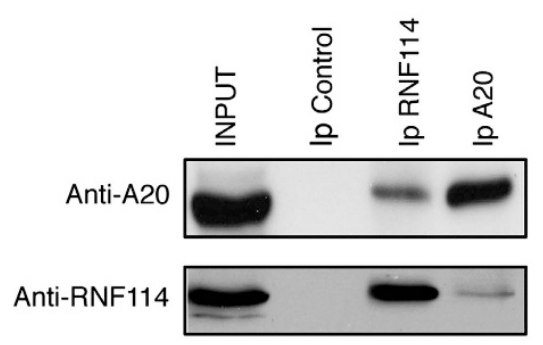

b
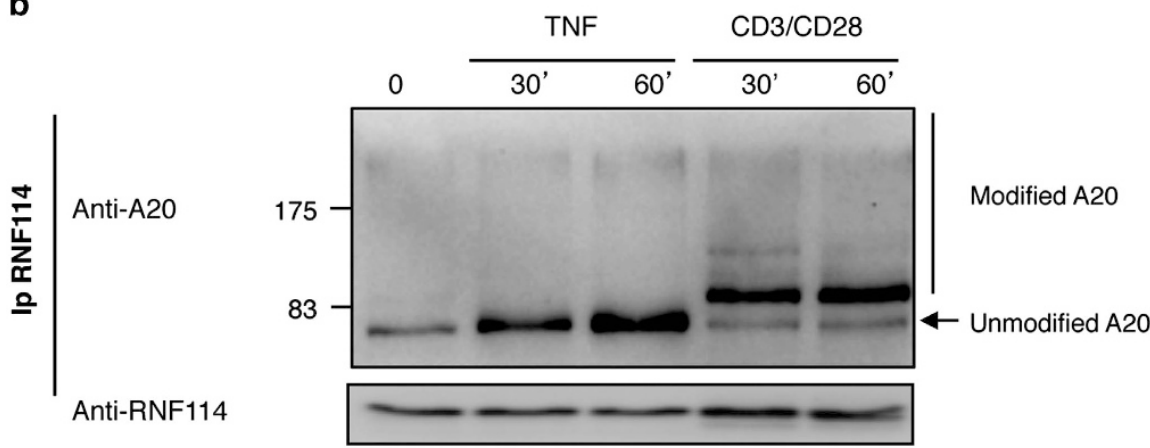

\begin{tabular}{l|l} 
上 & Anti-A20 \\
Anti-RNF114 & \\
Anti-IkB $\alpha$ \\
Charge Control
\end{tabular}

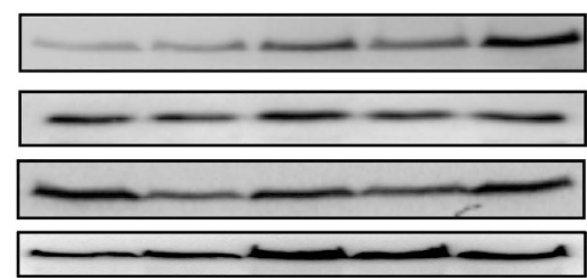

Figure 2 Interaction between endogenous RNF114 and A20 in T cells. (a) Jurkat T cells were used to confirm the endogenous interaction between the two proteins. Co-immunoprecipitations of A20 and RNF114, using anti-A20 and anti-RNF114 antibodies, are shown. (b) Co-immunoprecipitation between A20 and RNF114 in Jurkat T cells stimulated with TNF $\alpha$ or CD3/CD28 for the indicated times using anti-RNF114 antibodies 
a

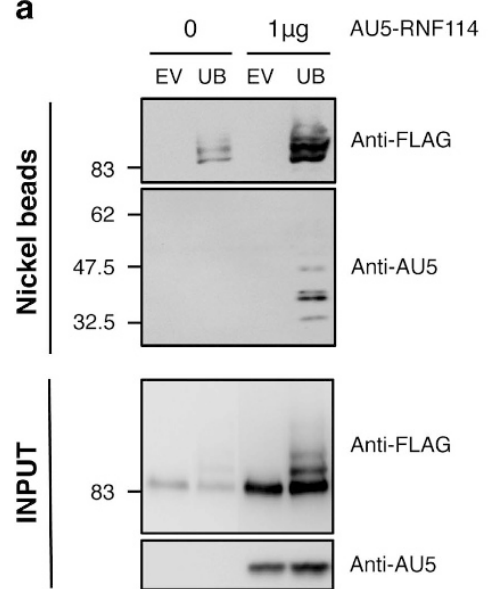

b

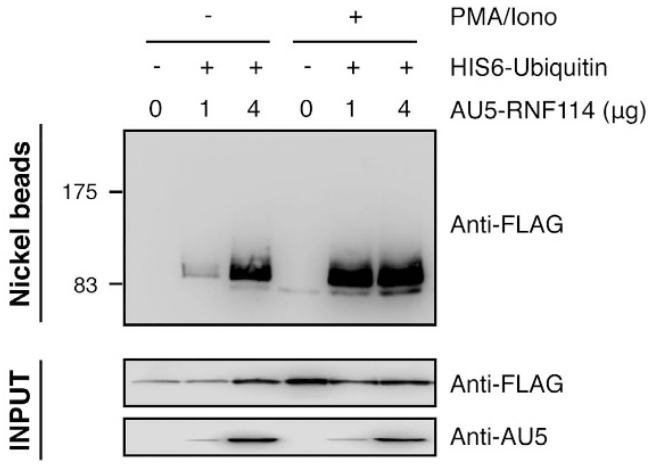

Figure 3 Effect of RNF114 on A20 ubiquitylation. (a) HEK293 cells were transfected with FLAG-A20WT in the presence or not of His6-ubiquitin and AU5-RNF114 as indicated. $\mathrm{His}_{6}$-ubiquitylated proteins were purified using denaturing conditions and $\mathrm{Ni}^{2+}$ chromatography. EV, empty vector; UB, His ${ }_{6}$-ubiquitin. (b) $\mathrm{HEK}^{2} 23$ cells were transfected with FLAG-A20WT in the presence or not of His6-ubiquitin and different amounts of AU5-RNF114 as indicated. Cells were stimulated for 30 min or not with PMA and ionomycin. His $_{6}$-ubiquitylated proteins were purified using denaturing conditions and $\mathrm{Ni}^{2+}$ chromatography

(Figure 3a). As TCR stimulation induced a modification of the molecular weight of A20-bound to RNF114, suggesting a post-translational modification of A20 (Figure 2b), we checked whether RNF114-induced A20 ubiquitylation was increased after phorbol 12-myristate 13-acetate (PMA)/ ionomycin in HEK293 cells. Our results revealed that level of ubiquitylated A20 increased in the presence of RNF114 and, interestingly, this effect is more pronounced after PMA/ ionomycin treatment, considered as a 'TCR-like' stimulus (Figure $3 b$ ). These results indicate that RNF114 promotes the ubiquitylation of A20.

RNF114 induces the stabilization of NF- $\kappa$ B regulators. To explore the role of RNF114 on NF- $k$ B pathway, we evaluated the effects of RNF114 overexpression on the stability of two NF- $\kappa$ B regulators, A20 and $I_{\kappa} \mathrm{B} \alpha$. We can observe on the blot (Figure 4a, left panel) as well as on the corresponding quantification (Figure $4 \mathrm{a}$, right panel) an accumulation of endogenous $I_{\kappa} \mathrm{B} \alpha$ and A20 levels in the presence of increasing amount of RNF114. The effect of RNF114 on $I_{\kappa} \mathrm{B} \alpha$ stability was also evaluated in a pulsechase experiment in the presence of $10 \mu \mathrm{g} / \mathrm{ml}$ of cycloheximide (CHX) (Figure 4b). We observed that $I_{\kappa} \mathrm{B} \alpha$ was better stabilized when low levels of RNF114 were expressed, suggesting that other possible targets could be affected when high doses of RNF114 are used. To confirm the implication of RNF114 on A20 and $\mathrm{I}_{\kappa} \mathrm{B} \alpha$ stabilities, we used a GFP-expressing lentiviral vector to transduce human Jurkat T cells with specific RNF114-shRNA. We used two different shRNA sequences against RNF114 (shRNF1 and shRNF2) to reduce possible off-target effects. The percentage of infection obtained was $\sim 90 \%$ for the two tested constructs, as well as for the control (Figure 4c, left panel) and the efficiency of endogenous RNF114 knockdown was confirmed by western blot (Figure 4c, right panel). When the expression of RNF114 was knocked down in Jurkat T cells, we observed a slight but consistent decrease of A20 and $I_{\kappa} \mathrm{B} \alpha$ expression. Corresponding western blot as well as its quantification is shown in Figure $4 \mathrm{~d}$. Altogether, the results presented here demonstrate that RNF114 plays a role in the regulation of $\mathrm{I}_{\kappa} \mathrm{B} \alpha$ and $\mathrm{A} 20$ stabilities. In addition, these results suggest that RNF114-induced A20 ubiquitylation would be responsible for its stabilization rather than its degradation.

Effect of RNF114 on NF- $\kappa$ B-dependent transcription. Because of the important role of A20 in the regulation of $\mathrm{NF}-\kappa \mathrm{B}$, we evaluated the effects of RNF114 on the function of this transcription factor. Luciferase assays were performed in both HEK293 (Figure 5a) and Jurkat T (Figure $5 b$ ) cells using the NF- $\kappa$ B reporter (3- $\kappa$ B enhancer ConA-luciferase plasmid). ${ }^{32}$ Overexpression of RNF114 significantly attenuated TNF-induced NF- $\kappa$ B activation in HEK293 and Jurkat T cells, as well as after TCR stimulation in Jurkat $T$ cells (Figures $5 a$ and $b$ ). To confirm the implication of RNF114 in the regulation of NF- $k \mathrm{~B}$, we used the previous Jurkat $T$ cells stably transduced with shRNF114 (Figure 4c). As can be observed in Figure 5c, knockdown of endogenous RNF114 enhanced TCR- as well as TNF $\alpha$-induced NF- $\kappa$ B activation in Jurkat $T$ cells. These results confirmed that RNF114 is a negative modulator of NF- $\kappa$ B transcription pathway acting through the stabilization of $\mathrm{A} 20$ and $\mathrm{I} \kappa \mathrm{B} \alpha$ inhibitors. The role of RNF114 might be important in a cellular context or situation where alternatives to moderate NF- $k \mathrm{~B}$ pathway could be required.

RNF114 is a regulator of TCR signaling. To determine whether RNF114 plays a role of modulator in T-cell function like its paralog TRAC1, knockdown experiments were performed using two shRNF114. First, Jurkat T cells were treated overnight with $\mathrm{TNF} \alpha(15 \mathrm{ng} / \mathrm{ml})$, known to induce T-cell apoptosis. Apoptosis events were evaluated by FACS analysis using Annexin $V$ and 7AAD staining (Figure 6a), as well as by western blot using anti-caspase 7 , caspase 9 or cleaved PARP antibodies (Figures $6 a$ and $b$ ). When cells 
a

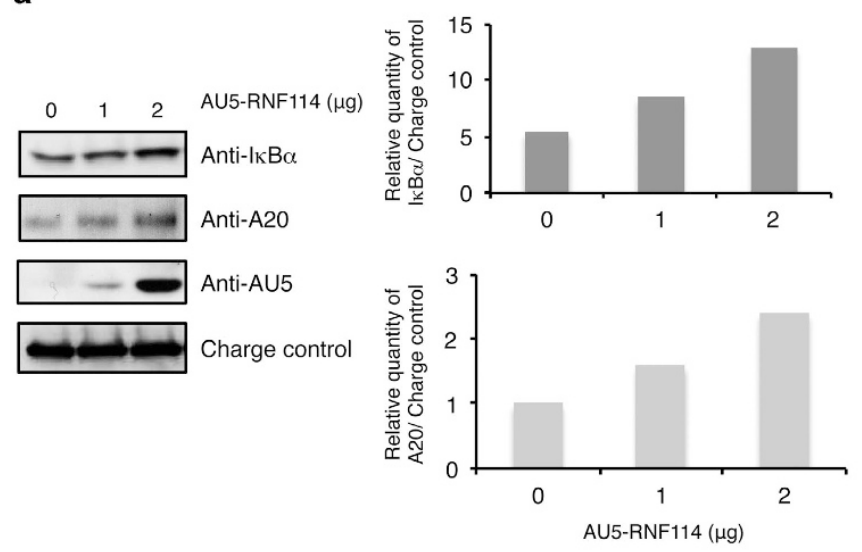

b

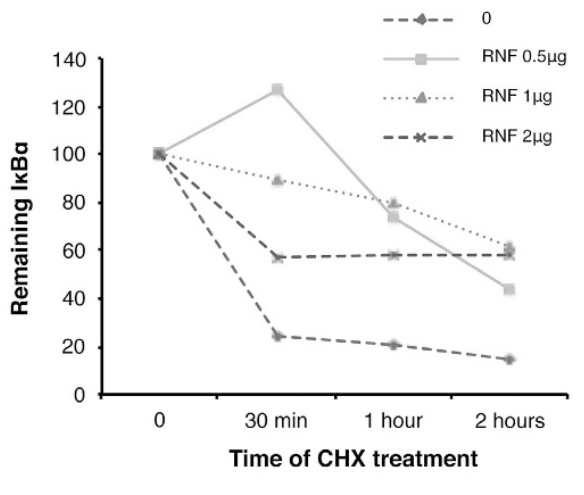

C
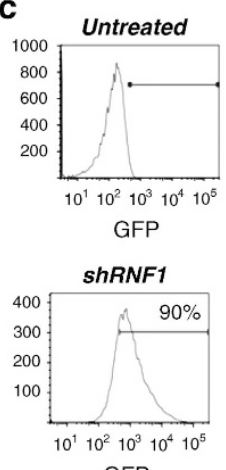

GFP

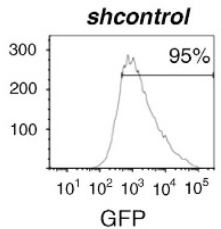

GFP

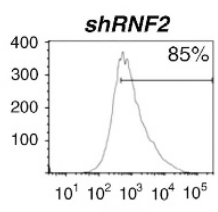

GFP

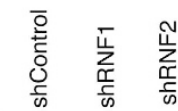

Anti-RNF114

Charge control
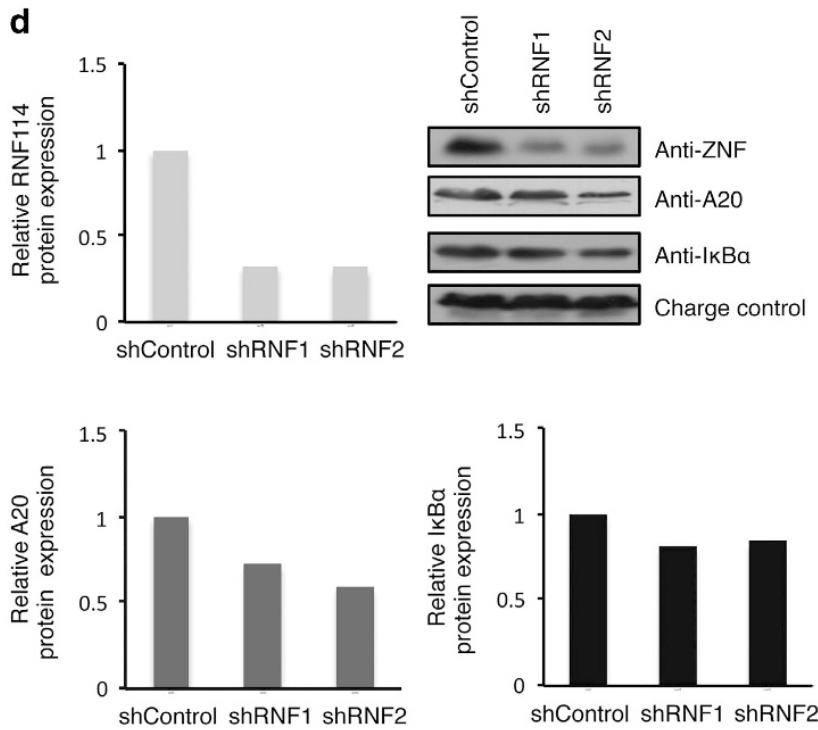

Figure 4 RNF114 increases the stability of both $I_{\kappa} B \alpha$ and A20. (a) HEK293 cells were transfected with different amounts of AU5-RNF114. Western blot analysis with anti-I $\mathrm{I}_{\kappa} \alpha,-\mathrm{A} 20,-\mathrm{AU} 5$ and $-\mathrm{GAPDH}$ (charge control) antibodies are presented. Graphs representing the relative amounts of $I_{\kappa} \mathrm{B} \alpha$ or $\mathrm{A} 20$ versus the charge control (GAPDH) of the western blots are shown. Representative result of different experiments is presented. (b) HEK293 cells were transfected with different amounts of RNF114 and treated for the indicated times with $\mathrm{CHX}(10 \mu \mathrm{g} / \mathrm{ml})$. A representative graph of three independent experiments is shown. (c) RNF114-specific gene silencing by lentivirus-mediated shRNA in Jurkat T cells. (Left panel) The percentage of infected cells was quantified by flow cytometry. (Right panel) Levels of protein expression were analyzed by western blot with the indicated antibodies. (d) Analysis of RNF114, A20 and $I_{\kappa} B \alpha$ protein expressions in Jurkat T cells transduced with shControl or shRNF1 and 2 . Western blot analysis with RNF114, A20 and $I_{\kappa} B \alpha$ antibodies are presented as well as a corresponding quantification. Representative result of different experiments is presented

were treated overnight with $\mathrm{TNF} \alpha$ alone, a reproducible inhibition of cell apoptosis was observed (Figure 6a). A mild but consistent effect is also observed on PARP or caspase7 and -9 cleavages when cells were treated for $6 \mathrm{~h}$ with both $\mathrm{TNF} \alpha$ and $\mathrm{CHX}$ (Figure 6b), indicating that RNF114 contributes to regulate T-cell apoptosis. However, RNF114 is not involved in the regulation of cell cycle in Jurkat $T$ cells (Figure 6c). Then, we checked the effect of RNF114 knockdown in the regulation of T-cell activation. For that purpose, Jurkat $T$ cells were treated overnight or not with CD3/CD28 antibodies or with PMA/ionomycin and stained with anti-CD69 and anti-CD25 antibodies (respectively) for FACS analysis. As shown in Figure 7, RNF114 knockdown induced a significant and reproducible increase of CD69 (Figure 7a) and CD25 (Figure 7b) expression, indicating that RNF114 is a negative regulator of T-cell activation.

\section{Discussion}

The regulation of the transcription factor $\mathrm{NF}-\kappa \mathrm{B}$ by posttranslational modifications with ubiquitin or ubiquitin-like proteins has been of increasing interest in recent years. $\mathrm{NF}-\kappa \mathrm{B}$ not only plays a crucial role in the regulation of immune and inflammatory responses, but also ensures basic functions during cell differentiation. In this study, we identified RNF114 as a new protein interacting with the inhibitor of NF- $\kappa \mathrm{B}, \mathrm{A} 20$. The domain involved in the interaction between the two proteins is present inside the E3 ligase domain of A20, suggesting that RNF114 takes part, as RNF11 or TAXBP1, in the A20 ubiquitin-editing complex. Moreover, overexpression or silencing experiments demonstrate that RNF114 is involved in the regulation of $\mathrm{NF}-\kappa \mathrm{B}$ activity. However, the inhibitory function of RNF114 on the TNF $\alpha$ - or TCR-induced $\mathrm{NF}-\kappa \mathrm{B}$ activation is not as strong as that mediated by $\mathrm{A} 20$ or $\mathrm{I} \kappa \mathrm{B} \alpha$ effects. The mild effects of RNF114 on NF- $\kappa \mathrm{B}$ activation 


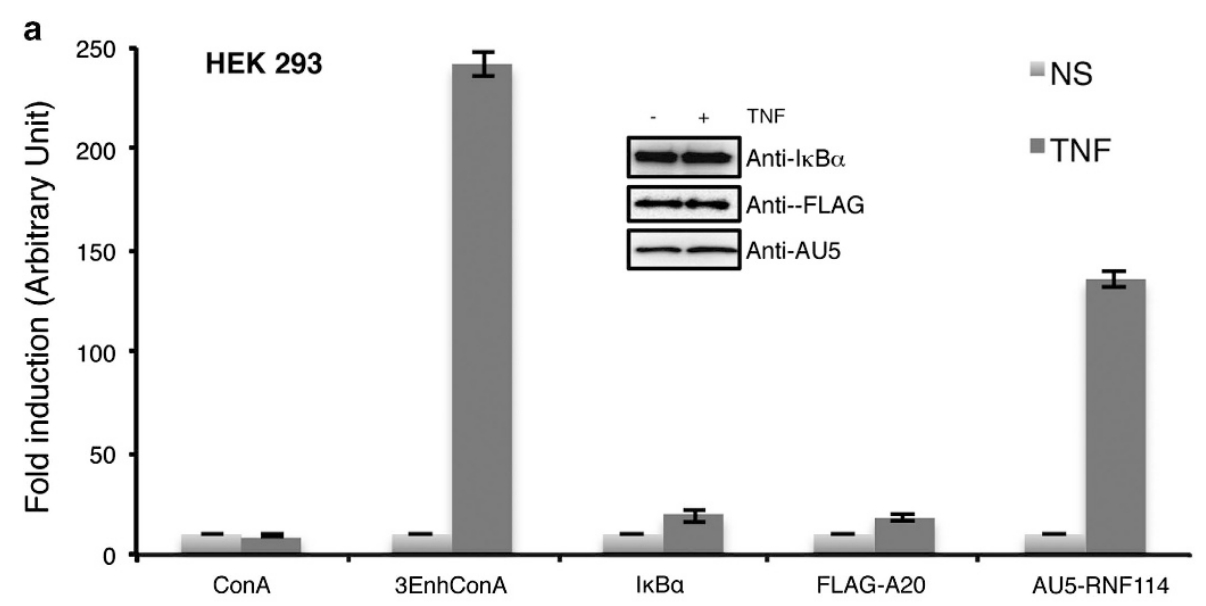

b

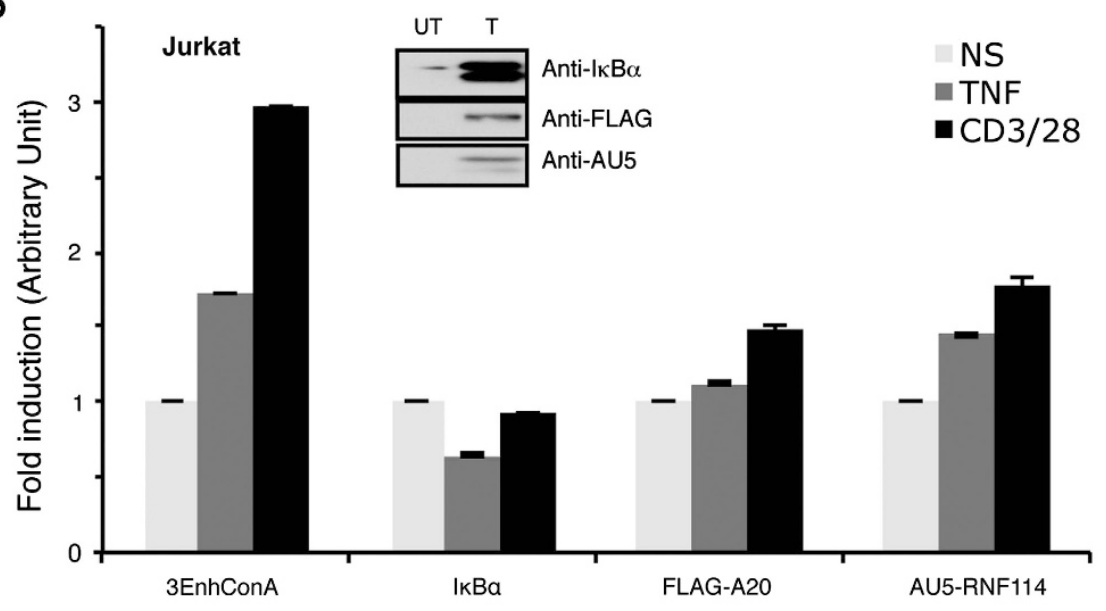

C
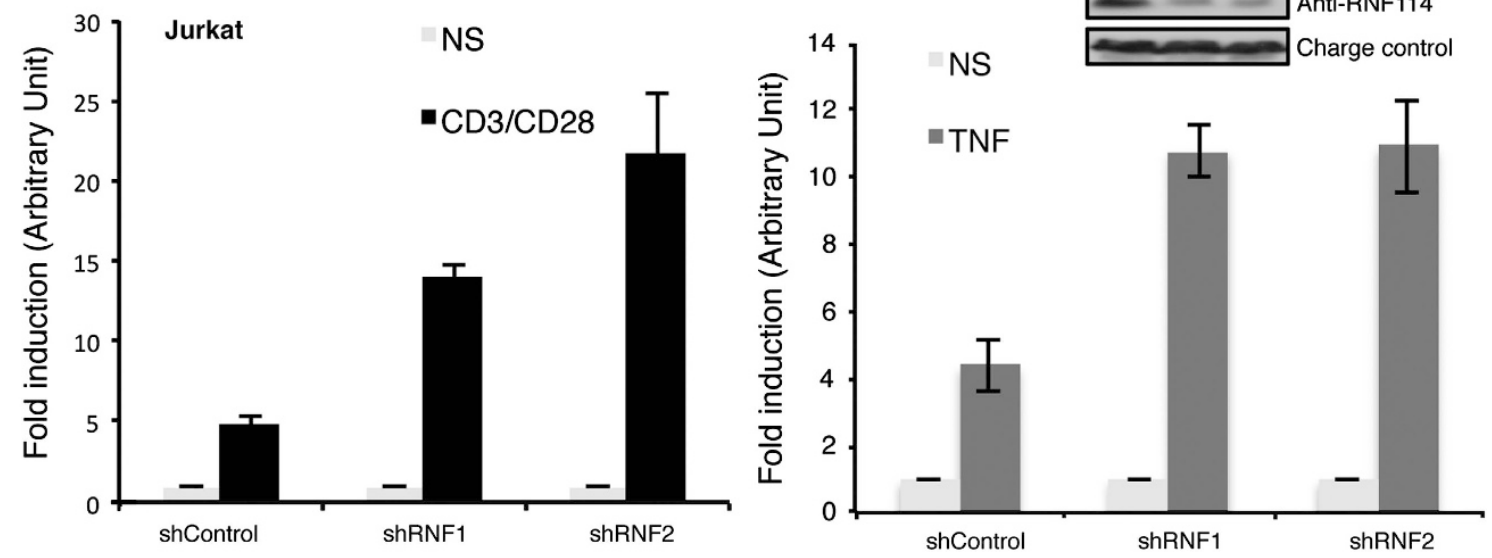

Figure 5 RNF114 is involved in the regulation of NF- $\kappa$ B activity. (a) Luciferase assay using the $3-\kappa B$ enhancer ConA-luciferase plasmid (3-EnhConA) in the presence of the indicated vectors in HEK293 cells stimulated or not with TNF $\alpha(5 \mathrm{~h})$. The graph represents the mean of three independent experiments done in triplicate. Levels of protein expression were analyzed by western blot with the indicated antibodies. (b) Luciferase assay using the 3-EnhConA reporter plasmids in the presence of the indicated vectors in Jurkat T cells stimulated for $5 \mathrm{~h}$ or not with TNF $\alpha$ or with the anti-CD3/CD28 antibodies. The graph represents the mean of three independent experiments done in triplicate. Levels of protein expression were analyzed by western blot with the indicated antibodies. (c) Luciferase assay using the 3-EnhConA reporter plasmids in Jurkat $\mathrm{T}$ cells stably transduced with shcontrol, shRNF1 or shRNF2 and stimulated or not with anti-CD3/CD28 antibodies (left panel) or with the TNF $\alpha$ (right panel). Each graph represents the mean of three independent experiments done in triplicate 

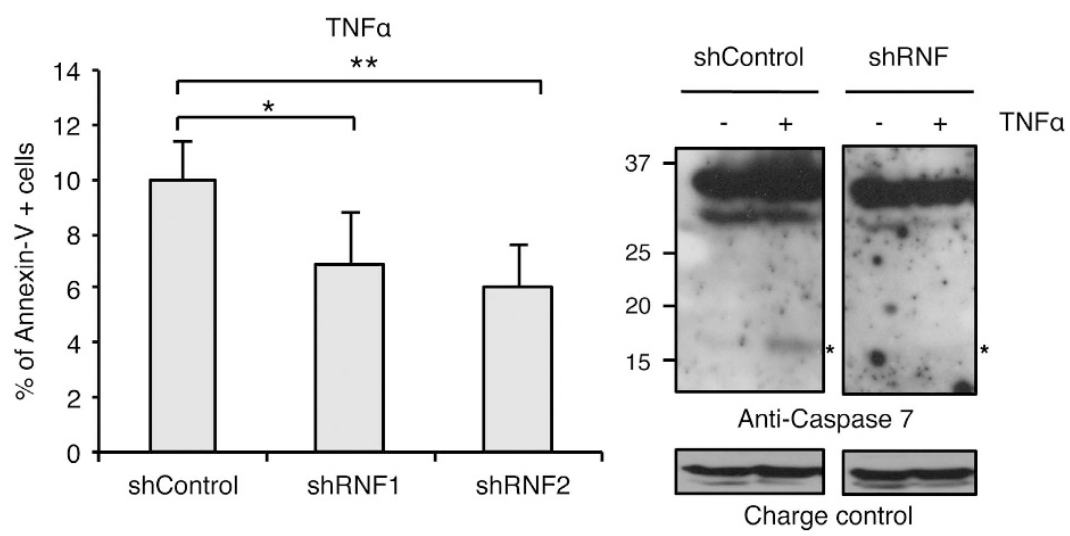

b

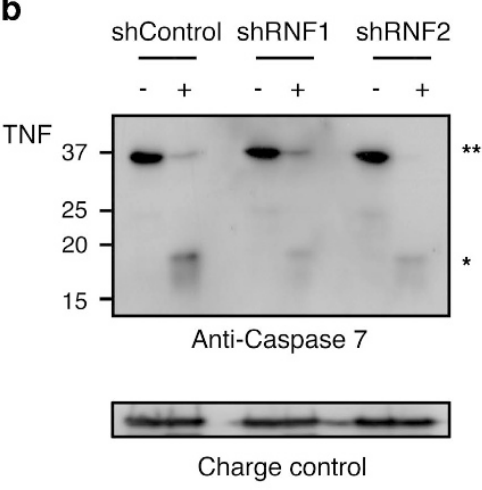

C

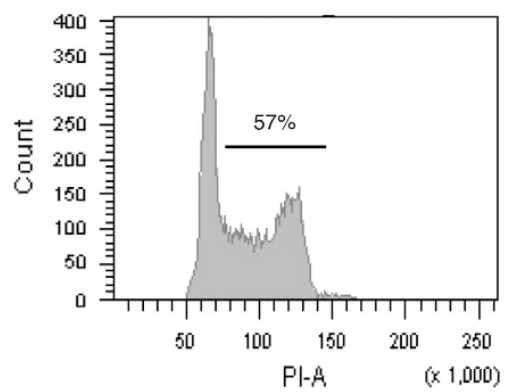

shControl shRNF1 shRNF2

shControl shRNF1 shRNF2

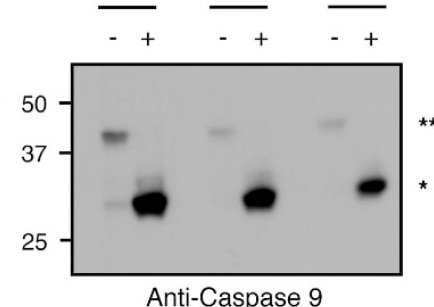

Anti-Caspase 9

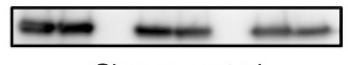

Charge control

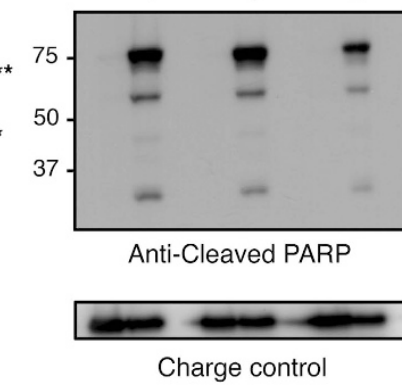

shRNF1
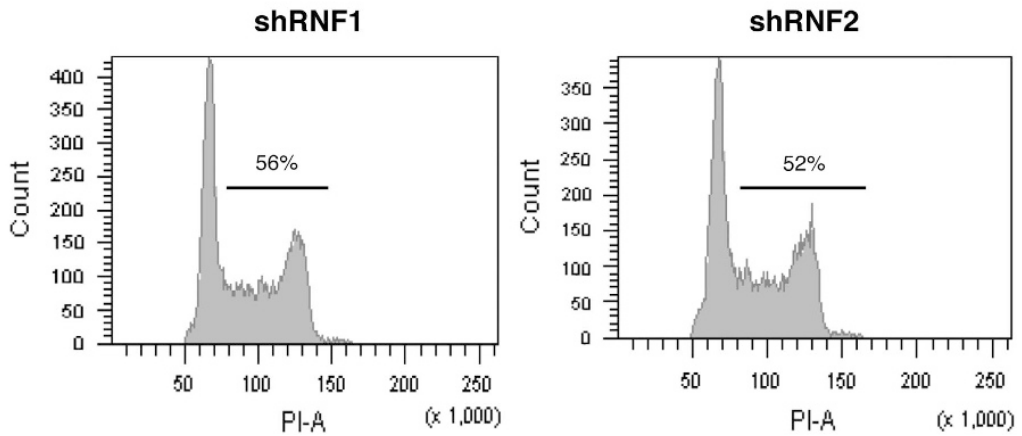

Figure 6 RNF114 does not modulate apoptosis or cell cycle in T cells. (a) Jurkat T cells stably transduced with shControl, shRNF114-1 or shRNF114-2 were treated overnight with TNF $\alpha(15 \mathrm{ng} / \mathrm{ml})$. Apoptosis was analyzed by FACS after Annexin V and 7AAD staining (the percentage of Annexin V-positive cells is shown) as well as by western blot analysis looking at the cleavage of caspase 7 induced after TNF $\alpha$ treatment (indicated by an asterisk * on the western blot). Data are expressed as the mean of three independent experiments ${ }^{*} P<0.05,{ }^{*} P<0.01$. (b) Jurkat T cells stably transduced with shControl, shRNF114-1 or shRNF114-2 were treated $6 \mathrm{~h}$ with a TNF $\alpha$ and CHX cocktail. Protein levels were analyzed by western blot with the indicated antibodies ("indicates cleaved form and ** indicates uncleaved form). (c) Cell cycle analysis of Jurkat $T$ cells transduced with shControl, shRNF114-1 or shRNF114-2 using propidium iodide staining and FACS analysis is shown

can be explained, at least in part, by the stabilization of A20 or $I_{\kappa} \mathrm{B} \alpha$ inhibitors. Others studies seem to highlight that RNF114 overexpression could have an activating effect on NF- $\kappa \mathrm{B}$ activity. ${ }^{33}$ This apparent discrepancy with our results might be because of experimental differences (different cell lines, stimuli, luciferase reporters) as well as different RNF114 expression levels. Indeed, we have evidences (data not shown) that the effect of RNF114 is dose dependent, indicating that regulation of its expression level or its posttranslational modification may also be important. In fact, we observed that RNF114 is also ubiquitylated (Figure 3a),
SUMOylated (data not shown) and stabilized/destabilized depending on its levels of expression (Figure $4 b$ ). Therefore, we hypothesize that RNF114 activity and function might be, like for A20, tissue and stimuli dependent.

Overexpression of RNF114 increases the stability of A20 and $\mathrm{I}_{\kappa} \mathrm{B} \alpha$ and could be the mechanism by which RNF114 regulates $\mathrm{NF}-\kappa \mathrm{B}$ pathway. A similar mechanism has recently been shown for the protein XAF1. ${ }^{28}$ However, in the case of A20, RNF114 overexpression increases A20 modification with ubiquitin without causing its degradation. Interestingly, the form of A20 bound to RNF114 in T cells 


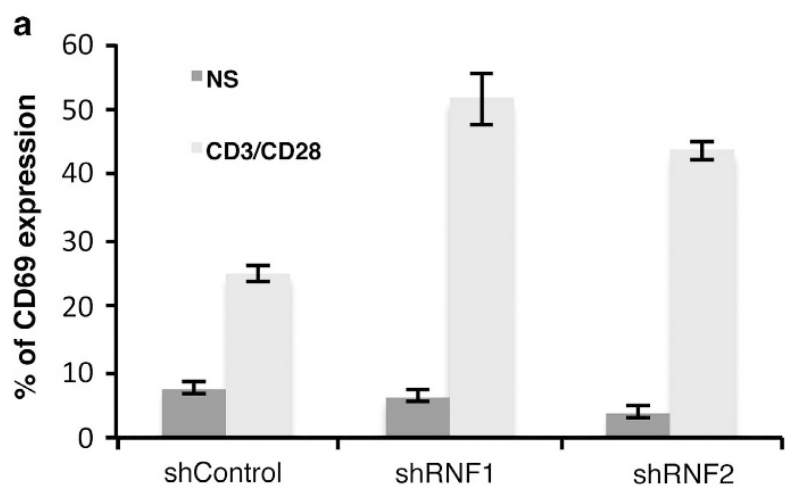

b
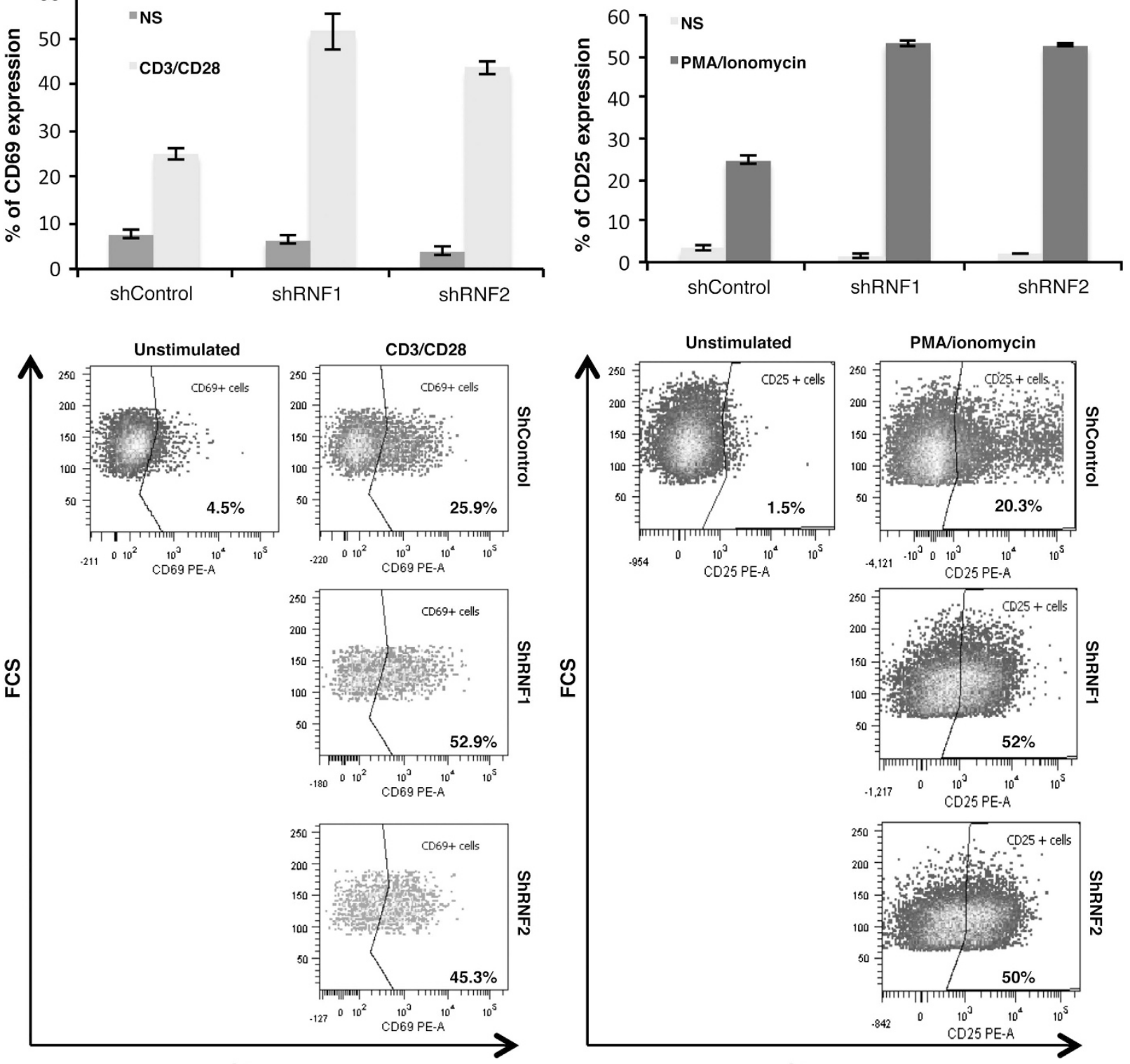

CD69

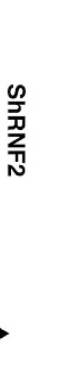

Figure 7 RNF114 is a regulator of TCR signaling. (a) Percentage of CD69-PE, obtained after FACS analysis, in Jurkat T cells stably transduced with shControl, shRNF114-1 or shRNF114-2 and stimulated or not overnight with CD3 and CD28 antibodies. A representative flow cytometry analysis of CD69 expression is shown. Background fluorescence values were set by use of isotype-matched irrelevant antibodies. (b) Percentage of CD25-PE, obtained after FACS analysis, in Jurkat T cells stably transduced with shControl, shRNF114-1 or shRNF114-2 and stimulated or not for $18 \mathrm{~h}$ with PMA/ionomycin. A representative flow cytometry analysis of CD25 expression is shown. Background fluorescence values were set by use of isotype-matched irrelevant antibodies

after TCR stimulation undergoes a mobility shift to a form with higher molecular weight, suggesting that under these conditions RNF114 can induce A20 modification and affect its activity.

Finally, we demonstrate that in T cells, RNF114 does not appear to be involved in the regulation of cell cycle, but rather in the regulation of T-cell apoptosis and activation as its knockdown induces a decrease of TNF $\alpha$-induced cell death and a significant increase of CD69 and CD25 expressions. Taken together, the results presented here show that RNF114 is a novel A20 interacting protein that is able to fine-tune NF- $\kappa \mathrm{B}$ activity in $\mathrm{T}$ cells stimulated with TNF $\alpha$ or anti-CD3/CD28 antibodies. RNF114 induces an increase of A20 ubiquitylation that in turn modifies A20 protein half-life. In T cells, RNF114 appears to be a modulator of $\mathrm{A} 2 \mathrm{O}$ function and of the NF- $\kappa \mathrm{B}$ activity required to regulate T-cell activation. Therefore, RNF114 represents a new target candidate to develop pharmaceutical strategies to control the activation of NF- $\kappa \mathrm{B}$ without suppressing its full capacity as a transcriptional activator. Studying the mechanisms that allow fine-tuning of this pathway represents an alternative to modulate immune and inflammatory 
responses without necessarily blocking other critical functions of NF- $\kappa$ B.

\section{Materials and Methods}

Cell culture and cell-based assays. HEK293 cells (ATCC, Manassas, VA, USA) were grown in DMEM (Gibco, Life Technologies, Carlsbad, CA, USA) and Jurkat cells (ATCC) in RPMI (Gibco), all supplemented with 10\% FBS and antibiotics. HEK293 cells were transfected using Lipofectamine (Invitrogen, Life Technologies) and Jurkat cells were transfected by electroporation (Bio-Rad, Life Sciences, Hercules, CA, USA) as previously described. ${ }^{34}$

To measure transcriptional activity, cells were co-transfected with a NF- $\kappa \mathrm{B}$ luciferase reporter plasmid $(3-\kappa B$ enhancer ConA-luciferase plasmid or 3-EnhConA ${ }^{32}$ ) together with plasmids expressing FLAG-A20, AU5-RNF114 or $I_{\kappa} \mathrm{B} \alpha$ Luciferase activity was measured as previously described. ${ }^{35}$

Cells were stimulated with either $10 \mathrm{ng} / \mathrm{ml}$ of TNF $\alpha$ (R\&D Systems, Minneapolis, MN, USA), with a mixture of CD3 (OKT3) and CD28 antibodies (BD Biosciences, Franklin Lakes, NJ, USA) or with PMA (20 ng/ml, SIGMA, St. Louis, MO, USA)/ionomycin (1 mM, Calbiochem, La Jolla, CA, USA) for the indicated times.

Depletion of endogenous RNF114 expression was achieved by RNA interference. The lentiviral shRNA expression plasmids were purchased from Open Biosystems (Thermo Scientific, Denver, CO, USA). Viral particles were produced as previously described by the Viral Vector Platform at Inbiomed Foundation. ${ }^{36}$ Jurkat T-cell transduction was carried out at a multiplicity of infection of 10 in order to achieve $100 \%$ infection. For the luciferase experiment, cells were transfected with a NF- $\kappa \mathrm{B}$ luciferase reporter plasmid (3- $\kappa$ B enhancer ConA-luciferase plasmid) and luciferase activity was measured later. ${ }^{32}$

Flow cytometry. For cell cycle analysis, Jurkat cells were washed with cold PBS and fixed with $70 \%$ ethanol overnight. Cells were then washed twice with PBS and resuspended in PBS containing $5 \mathrm{mg} / \mathrm{ml}$ propidium iodide $(\mathrm{PI})$ and $10 \mu \mathrm{g} / \mathrm{ml}$ RNase A (Sigma-Aldrich, St. Louis, MO, USA). Cell cycle analysis was performed on GFP (530/30BP)-positive and alive cells, excluding doublets.

To track $T$ cells undergoing apoptosis, Jurkat cells were treated overnight with $\mathrm{TNF} \alpha(15 \mathrm{ng} / \mathrm{ml}, \mathrm{R} \& \mathrm{D})$ alone or for $6 \mathrm{~h}$ in combination with $\mathrm{CHX}(10 \mu \mathrm{g} / \mathrm{ml}, \mathrm{SIGMA})$ Co-staining with Annexin-V-PE (BD Biosciences; 585/42BP) and 7-aminoactinomycin D (7AAD, SIGMA; $670 \mathrm{LP})$ was performed to differentiate early and late apoptosis as well as necrotic cells. The percentage of each population was analyzed by flow cytometry gated on GFP (530/30BP)-positive cells, excluding doublets.

For activation assays, $T$ cells were cultured as described above and stimulated for $18 \mathrm{~h}$ with anti-CD3 and anti-CD28 antibodies or PMA/ionomycin to check respectively CD69 and CD25 expression. CD69-PE (BD Biosciences; 585/42BP and CD25-PE (ImmunoStep, Salamanca, Spain; 585/42BP) expressions were measured by FACS, gated on GFP-positive cells, excluding doublets and dead cells.

Data represent the mean of three independent experiments done in triplicate. A total of $10^{4}$ events were counted for each sample. Data were collected on a FACSCanto (BD Biosciences) and were analyzed using FlowJo software (www.flowjo.com)

Immunodetections. Western blot detections were performed with the following primary antibodies: FLAG (SIGMA), AU5 (Covance, Princetown, NJ, USA), A20 (Calbiochem), I $\kappa$ B $\alpha$ (Cell Signaling Technology, Beverley, MA, USA), GAPDH (SIGMA) and ZNF313/RNF114 (Santa Cruz Biotechnology, Santa Cruz, CA, USA), Sam68 (Santa Cruz Biotechnology), caspase 7 (Cell Signaling Technology), Cleaved-PARP (Cell Signaling Technology) and Caspase 9 (Cell Signaling Technology).

Co-immunoprecipitation experiments were performed using Protein-G crosslinked with $4 \mu \mathrm{g}$ of antibody/point of FLAG, AU5, A20 or RNF114 antibodies to immunoprecipitate exogenous or endogenous proteins as indicated. In all cases, cells were lysed for $15 \mathrm{~min}$ on ice in $50 \mathrm{mM}$ sodium fluoride, $5 \mathrm{~mm}$ tetra-sodium pyrophosphate, $10 \mathrm{~mm} \beta$-glyceropyrophosphate, $1 \%$ Igepal CA-630, $2 \mathrm{mM}$ EDTA, $20 \mathrm{~mm} \mathrm{Na} \mathrm{HPO}_{4}, 20 \mathrm{~mm} \mathrm{NaH} \mathrm{PO}_{4}$ and $1.2 \mathrm{mg} / \mathrm{ml}$ Complete protease inhibitor cocktail (Roche, Indianapolis, IN, USA). His $s_{6}$-ubiquitylated or SUMOylated proteins were purified using denaturing conditions and $\mathrm{Ni}^{2+}$ chromatography as previously described. $^{37}$

Cloning. A plasmid encoding human ZNF313/RNF114 cDNA (RZPD) and PCR-subcloning was performed into pGEX6 and AU5 plasmids. The A20 gene was amplified from cDNA of purified T cells using the following primers $5^{\prime}$-ACAAA
CGAATTCATGGCTGAAGTCCTTC-3' and 5'-GCCGAGGAATTCTTAGGGGCAG TTGGGCGTTTC- $3^{\prime}$ and cloned into a PCEFL FLAG vector. Primer sequences for deletion constructs are available upon request. The accuracy of all cloning and mutagenesis procedures was verified by sequencing.

All experiments presented in this manuscript were done at least in triplicate. For luciferase experiments and FACS analysis, data represent the mean of at least three independent experiments done in triplicate.

\section{Conflict of Interest}

The authors declare no conflict of interest.

Acknowledgements. We thank the platform managers Dr Cristina Sanchez for her help in viral production and Lorea Zabaleta for her technical support. Our group is supported by 'Obra Social Kutxa', Diputación Foral de Gipuzkoa and Saiotek program from the Department of Industry, Tourism and Trade of the Government of the Autonomous Community of the Basque Country. This work was also funded by the Ramón y Cajal Program, Ministerio de Educación y Ciencia grant BFU2011-28536 and BFU2006-12991, Fondo de Investigaciones Sanitarias (FIS) CIBERhed.

1. Gilmore TD. Introduction to NF-kappaB: players, pathways, perspectives. Oncogene 2006; 25: 6680-6684.

2. DiDonato JA, Mercurio F, Karin M. NF-kappaB and the link between inflammation and cancer. Immunol Rev 2012; 246: 379-400.

3. Hayden MS, Ghosh S. Shared principles in NF-kappaB signaling. Cell 2008; 132: 344-362.

4. Perkins ND. The Rel/NF-kappa B family: friend and foe. Trends Biochem Sci 2000; 25: 434-440.

5. Perkins ND, Gilmore TD. Good cop, bad cop: the different faces of NF-kappaB. Cell Death Differ 2006; 13: 759-772.

6. Vereecke L, Beyaert R, van Loo G. The ubiquitin-editing enzyme A20 (TNFAIP3) is a central regulator of immunopathology. Trends Immunol 2009; 30: 383-391.

7. Oeckinghaus A, Ghosh S. The NF-kappaB family of transcription factors and its regulation. Cold Spring Harb Perspect Biol 2009; 1: a000034.

8. Jono H, Lim JH, Chen LF, Xu H, Trompouki E, Pan ZK et al. NF-kappaB is essential for induction of CYLD, the negative regulator of NF-kappaB: evidence for a novel inducible autoregulatory feedback pathway. J Biol Chem 2004; 279: 36171-36174.

9. Renner F, Schmitz ML. Autoregulatory feedback loops terminating the NF-kappaB response. Trends Biochem Sci 2009; 34: 128-135

10. Shembade N, Harhaj EW. Regulation of NF-kappaB signaling by the A20 deubiquitinase. Cell Mol Immunol 2012; 9: 123-130.

11. Beyaert R, Heyninck K, Van Huffel S. A20 and A20-binding proteins as cellular inhibitors of nuclear factor-kappa B-dependent gene expression and apoptosis. Biochem Pharmacol 2000; 60: 1143-1151.

12. Lee EG, Boone DL, Chai S, Libby SL, Chien M, Lodolce JP et al. Failure to regulate TNF-induced NF-kappaB and cell death responses in A20-deficient mice. Science 2000; 289: 2350-2354.

13. Boone DL, Turer EE, Lee EG, Ahmad RC, Wheeler MT, Tsui C et al. The ubiquitinmodifying enzyme A20 is required for termination of Toll-like receptor responses. Nat Immunol 2004; 5: 1052-1060.

14. Verstrepen L, Verhelst K, van Loo G, Carpentier I, Ley SC, Beyaert R. Expression, biological activities and mechanisms of action of A20 (TNFAIP3). Biochem Pharmacol 2010; 80: 2009-2020.

15. Coornaert B, Carpentier I, Beyaert R. A20: central gatekeeper in inflammation and immunity. J Biol Chem 2009; 284: 8217-8221.

16. Shembade N, Harhaj NS, Liebl DJ, Harhaj EW. Essential role for TAX1BP1 in the termination of TNF-alpha-, IL-1- and LPS-mediated NF-kappaB and JNK signaling. EMBO J 2007; 26: 3910-3922

17. Shembade N, Parvatiyar K, Harhaj NS, Harhaj EW. The ubiquitin-editing enzyme A20 requires RNF11 to downregulate NF-kappaB signalling. EMBO J 2009; 28: 513-522.

18. Shembade N, Harhaj NS, Parvatiyar K, Copeland NG, Jenkins NA, Matesic LE et al. The E3 ligase Itch negatively regulates inflammatory signaling pathways by controlling the function of the ubiquitin-editing enzyme A20. Nat Immunol 2008; 9: 254-262.

19. Coornaert B, Baens M, Heyninck K, Bekaert T, Haegman M, Staal J et al. T cell antigen receptor stimulation induces MALT1 paracaspase-mediated cleavage of the NF-kappaB inhibitor A20. Nat Immunol 2008; 9: 263-271.

20. Dorronsoro A, Lang V, Jakobsson E, Ferrin I, Salcedo JM, Fernandez-Rueda J et al. Identification of the NF-kappaB inhibitor A20 as a key regulator for human adipogenesis. Cell Death Dis 2013; 4: e972.

21. Vereecke L, Beyaert R, van Loo G. Genetic relationships between A20/TNFAIP3, chronic inflammation and autoimmune disease. Biochem Soc Trans 2011; 39: 1086-1091. 
22. Stuart PE, Nair RP, Ellinghaus E, Ding J, Tejasvi T, Gudjonsson JE et al. Genome-wide association analysis identifies three psoriasis susceptibility loci. Nat Genet 2010; 42 : 1000-1004.

23. Nair RP, Duffin KC, Helms C, Ding J, Stuart PE, Goldgar D et al. Genome-wide scan reveals association of psoriasis with IL-23 and NF-kappaB pathways. Nat Genet 2009; 41 : 199-204.

24. Zhao H, Li CC, Pardo J, Chu PC, Liao CX, Huang J et al. A novel E3 ubiquitin ligase TRAC-1 positively regulates T cell activation. J Immunol 2005; 174: 5288-5297.

25. Giannini AL, Gao Y, Bijlmakers MJ. T-cell regulator RNF125/TRAC-1 belongs to a novel family of ubiquitin ligases with zinc fingers and a ubiquitin-binding domain. Biochem $\mathrm{J} 2008$; 410: 101-111.

26. Li N, Sun $\mathrm{H}$, Wu Q, Tao D, Zhang S, Ma Y. Cloning and expression analysis of a novel mouse zinc finger protein gene Znf313 abundantly expressed in testis. J Biochem Mol Biol 2007; 40: 270-276

27. Ma YX, Zhang SZ, Hou YP, Huang XL, Wu QQ, Sun Y. Identification of a novel human zinc finger protein gene ZNF313. Sheng Wu Hua Xue Yu Sheng Wu Wu Li Xue Bao (Shanghai) 2003; 35: 230-237.

28. Han J, Kim YL, Lee KW, Her NG, Ha TK, Yoon S et al. ZNF313 is a novel cell cycle activator with an E3 ligase activity inhibiting cellular senescence by destabilizing p21(WAF1.). Cell Death Differ 2013; 20: 1055-1067.

29. Vincenz C, Dixit VM. 14-3-3 proteins associate with A20 in an isoform-specific manner and function both as chaperone and adapter molecules. J Biol Chem 1996; 271: 20029-20034.

30. Heyninck K, De Valck D, Vanden Berghe W, Van Criekinge W, Contreras R, Fiers W et al. The zinc finger protein A20 inhibits TNF-induced NF-kappaB-dependent gene expression by interfering with an RIP- or TRAF2-mediated transactivation signal and directly binds to a novel NF-kappaB-inhibiting protein ABIN. J Cell Biol 1999; 145: 1471-1482.

31. De Valck D, Heyninck K, Van Criekinge W, Contreras R, Beyaert R, Fiers W. A20, an inhibitor of cell death, self-associates by its zinc finger domain. FEBS Lett 1996; $\mathbf{3 8 4}$ $61-64$.
32. Rodriguez MS, Thompson J, Hay RT, Dargemont C. Nuclear retention of IkappaBalpha protects it from signal-induced degradation and inhibits nuclear factor kappaB transcriptional activation. J Biol Chem 1999; 274: 9108-9115.

33. Bijlmakers MJ, Kanneganti SK, Barker JN, Trembath RC, Capon F. Functional analysis of the RNF114 psoriasis susceptibility gene implicates innate immune responses to double-stranded RNA in disease pathogenesis. Hum Mol Genet 2011; 20: 3129-3137.

34. Lang V, Semichon M, Michel F, Brossard C, Gary-Gouy H, Bismuth G. Fyn membrane localization is necessary to induce the constitutive tyrosine phosphorylation of Sam68 in the nucleus of T lymphocytes. J Immunol 1999; 162: 7224-7232.

35. Rodriguez MS, Wright J, Thompson J, Thomas D, Baleux F, Virelizier JL et al. Identification of lysine residues required for signal-induced ubiquitination and degradation of I kappa B-alpha in vivo. Oncogene 1996; 12: 2425-2435.

36. Carcamo-Orive I, Gaztelumendi A, Delgado J, Tejados N, Dorronsoro A, FernandezRueda $\mathrm{J}$ et al. Regulation of human bone marrow stromal cell proliferation and differentiation capacity by glucocorticoid receptor and AP-1 crosstalk. J Bone Miner Res 2010; 25: 2115-2125.

37. Tatham MH, Rodriguez MS, Xirodimas DP, Hay RT. Detection of protein SUMOylation in vivo. Nat Protoc 2009; 4: 1363-1371.

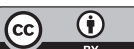

Cell Death and Disease is an open-access journal published by Nature Publishing Group. This work is licensed under a Creative Commons Attribution 3.0 Unported License. The images or other third party material in this article are included in the article's Creative Commons license, unless indicated otherwise in the credit line; if the material is not included under the Creative Commons license, users will need to obtain permission from the license holder to reproduce the material. To view a copy of this license, visit http://creativecommons.org/licenses/by/3.0/ 